\title{
A Review on Prerequisites of a Set-Up for Particle Detection by Ultrasonic Waves in Aluminium Melts
}

\author{
Mertol Gökelma, Damien Latacz, Bernd Friedrich \\ IME Process Metallurgy and Metal Recycling, RWTH Aachen University, Aachen, Germany \\ Email: mgoekelma@ime-aachen.de
}

Received 20 January 2016; accepted 21 March 2016; published 24 March 2016

Copyright (C) 2016 by authors and Scientific Research Publishing Inc.

This work is licensed under the Creative Commons Attribution International License (CC BY). http://creativecommons.org/licenses/by/4.0/

(c) (i) Open Access

\begin{abstract}
In many applications in aluminium industry, the number of inclusion-critical products increases and the quality of those products depend on the inclusion concentration and size. In order to improve the quality of aluminium products and the effectiveness of the processes, a reliable and cheaper on-line detection method is needed. Ultrasonic detection has been used in steel industry, but relatively rare in aluminium industry, although it would theoretically allow for an online non-intrusive detection of the cleanliness of the melt. In this work, the current information on ultrasonic inclusion detection was gathered and recommendations were provided on the Prerequisites for a set-up for ultrasonic detection of non-metallic inclusions in aluminium as a contribution on previous works. It has been concluded that ultrasonic waves seem promising, and should be experimented more on an industrial level to have a clear view on the potentials of the method.
\end{abstract}

\section{Keywords}

Non-Metallic Particles, Ultrasonic Waves, Aluminium, Online Detection

\section{Introduction}

Aluminium is widely present in daily life, being used in transportation, packaging, electrical engineering and many other fields. In many applications, the number of inclusion-critical products increases and the quality of those products depends on the inclusion concentration and size. The non-metallic inclusions impact the formability and surface quality crucially. This issue makes the detection of the inclusions a topic of interest [1]-[3].

Several inclusion detection methods exist, such as PoDFA (Porous Disc Filtration Analysis), PreFil (Pressure Filtration Melt Cleanliness Analyser) as offline methods and LiMCA (Liquid Metal Cleanliness Analyser) and 
Metalvision MV20/20 as online methods. The PoDFA and PreFil techniques are based on concentration of the solid inclusions by filtering the liquid sample. The filter cake is later examined by metallographically. It has the advantages of providing good information about the shapes and types but it lacks of a global knowledge of the concentration of the melt and the evaluation is a skilled and a long operation [1] [2] [4] [5]. The LiMCA method measures the concentration and size of the solid inclusions entering in a small orifice through which the liquid is forced to pass. The measurement is performed by detecting the voltage difference between two electrodes which are located in both sides of the orifice. Despite having the advantage of being an on-line detection method (semi real time), the LiMCA system usually detects particles in the range of 15 to $150 \mu \mathrm{m}$. It has difficulties by detecting smaller particles, and information about the shape of inclusions or their global concentration in the melt is not possible to obtain since the sampling volume is only $6 \mathrm{ml}$. Moreover, it is also an expensive system [2] [6]. The detection methods are summarized in Table 1.

\section{Motivation}

In order to improve the quality of aluminium products and the effectiveness of the processes, a reliable and cheaper on-line detection method is needed [9]. Ultrasonic detection has been used in steel industry, but relatively rare in aluminium industry, although it would theoretically allow for an online non-intrusive detection of the cleanliness of the melt [1] [4] [5] [7] [8] [10]-[12]. An on-line detection system for aluminium called Metal Vision MV20/20 is available, but has some disadvantages as its price or inability to differentiate small particles. This method will be discussed in detail later.

This work aims gathering the current information on ultrasonic inclusion detection and providing recommendations on the requirements for an ultrasonic detection set-up for aluminium industry as a contribution on previous works.

\section{Background of the Ultrasonics}

Ultrasounds are defined as waves with frequency higher than $20 \mathrm{kHz}$. It consists of a series of mechanical compressions and expansions in a system. They can be created by a piezoelectric transducer: an apparatus that converts electric energy into mechanical energy [13].

In metallurgical applications, a few parameters will be of importance. First of all, velocity, as it may be used to determine the position of inclusions in the melt by measuring the delay between emission and reception. The velocity of the wave is determined by:

$$
V=f \times \lambda=\sqrt{\frac{c_{i j}}{\rho}}
$$

where $V$ is the velocity of the wave in $\mathrm{cm} / \mathrm{ms}, f$ frequency, $\lambda$ wavelength, $C_{i j}$ its elastic constant with $i$ and $j$ representing respectively the longitudinal and shear direction, and $\rho$ density [13]. An approximate value for longitudinal velocity of the sonic waves in solid aluminium is $0.632 \mathrm{~cm} / \mathrm{ms}$ [14].

Secondly, the attenuation of the amplitude of the wave must be taken into account. As it will be explained in the next chapter, detection works by getting signals echoing back from the inclusions, and thus a high enough amplitude is needed for the detection of this return signal. The amplitude attenuation follows this equation:

$$
A=A_{0} e^{-\alpha x}
$$

where $A_{0}$ is the initial amplitude, $x$ the distance from initial location and $\alpha$ the attenuation coefficient of the

Table 1. Comparison of on-line and off-line detection methods in aluminium melts [7] [8].

\begin{tabular}{ccccc}
\hline & Technique & Real Time & Sample Volume & Detection Size Range $(\mu \mathrm{m})$ \\
\hline PoDFA & Metallography & No & Medium & None \\
PreFil & Metallography & Partial & Medium & None \\
LiMCA & Change in Electrical Resistivity & Semi & Small & $15-150$ \\
MetalVision & Ultrasound & Yes & Large & $20-160$ \\
\hline
\end{tabular}


medium in which the waves is propagating. The coefficient $\alpha$ is dependent on the frequency and thus can be properly determined only by experimenting in the used medium [14] [15].

Thirdly, the acoustic impedance $Z$ is used to determine the acoustic transmission and reflection at the interface between two materials. It has primary importance in an ultrasonic detection system to determine the efficiency of the system at the interface between the rods and the aluminium melt. The acoustic impedance is defined as follow [13].

$$
Z=\rho \times V
$$

The amount of energy reflected at the interface can be defined as a ratio $R$ of the initial energy by the following equation:

$$
R=\left(\frac{Z_{2}-Z_{1}}{Z_{2}+Z_{1}}\right)^{2}
$$

where; $Z_{1}$ and $Z_{2}$ are respectively the acoustic impedance of the first and second material.

The power variation can be of great order and this must absolutely be taken into account when choosing the emitting power. It is worth noticing that as medium of propagation changes, the velocity will also change, but as the frequency is constant, the wavelength will also change in the second material [15]. An illustration of the transmission principle can be seen in Figure 1.

Ultrasounds have currently many uses, such as; echography, degassing of Aluminum, filtration of chemical compounds, motion sensors.

\section{Applied Ultrasonic Methods for Aluminium}

The use of ultrasonic waves allows for a non-invasive and non-destructive treatment of the melt during processes. From the metallurgical point of view, improving the current techniques by the use of ultrasonic waves can greatly improve the efficiency of the detection processes. Ultrasonic detection of particles would allow for a fast and online detection, saving adjustment time and costs.

\subsection{Metal Vision MV20/20 Ultrasonic Inclusion Analyzer}

The MetalVision MV20/20 detector is composed of two buffer rods, one emitting and one receiving the waves, a reflector located in the melt, as shown in Figure 2, and a computer for display of the information [16].

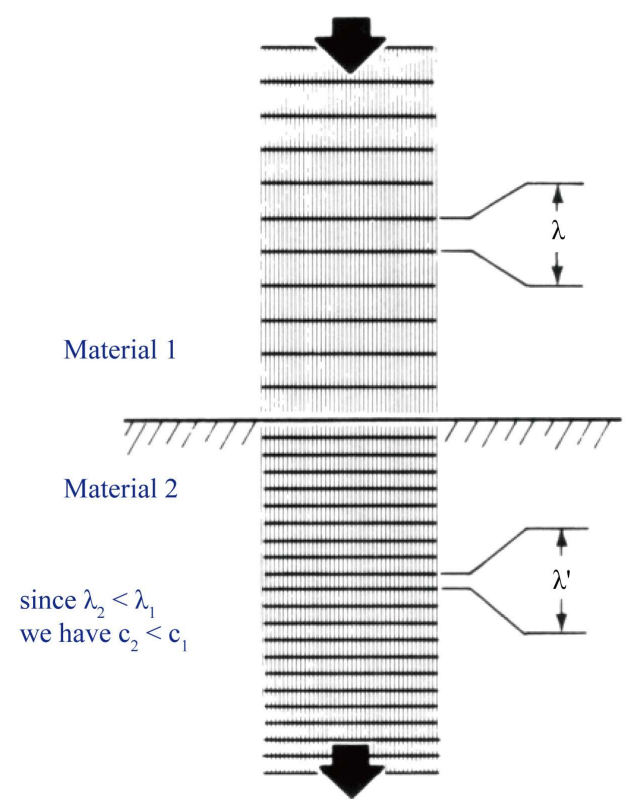

Figure 1. Transmission of waves between two materials [15]. 
MetalVision uses the principle of attenuation and reflection of a signal, correlating it with the particle size distribution and the concentration of inclusions in the melt [17]. It provides continuous information about the cleanliness of the melt. The system emits 100 pulse of $250 \mathrm{MHz}$ frequency per second [7]. The minimum size of a particle detectable by MetalVision is $20 \mu \mathrm{m}$ and goes up to $160 \mu \mathrm{m}$ [16].

The system provides the user with three plots: cleanliness value, particle size and particle count for each particle size range, as shown in Figure 3. A cleanliness value of around 100 indicates a melt containing relatively few particles, as a value around 0 indicates a rather large amount of particles. It is a general indication of the inclusion concentration of the melt, regardless of their size [18].

MetalVision has advantages, as it is able to detect particle up to $20 \mu \mathrm{m}$, but it lacks in constant results and is dependent on the concentration change of the biggest particles. This could be a direction of improvement while developing an ultrasonic detection system.

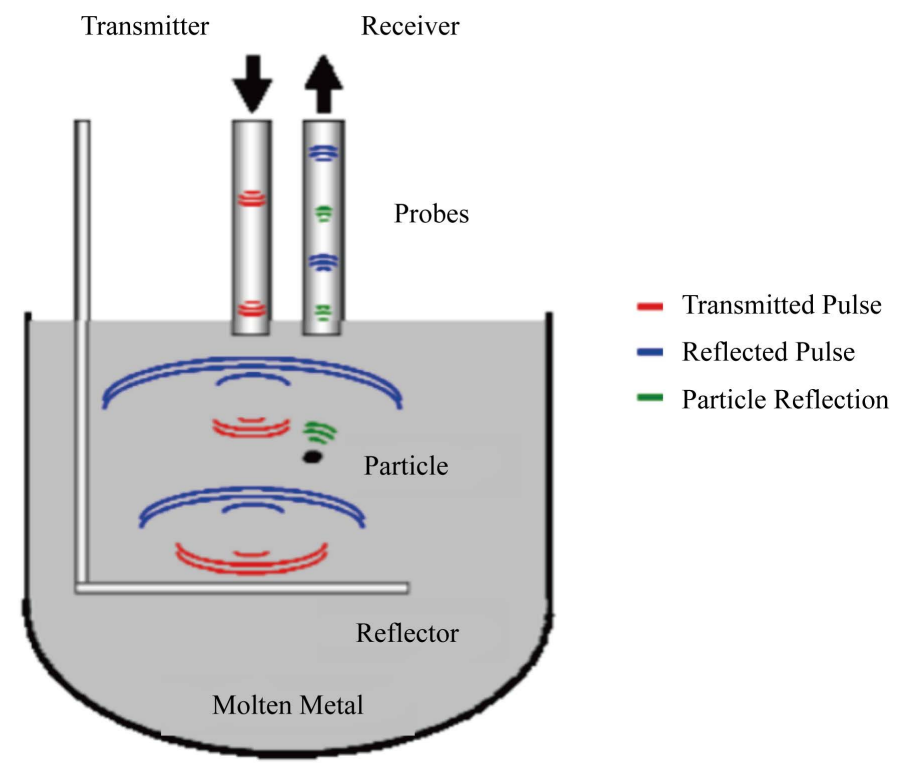

Figure 2. Particle detection principle of MetalVision.

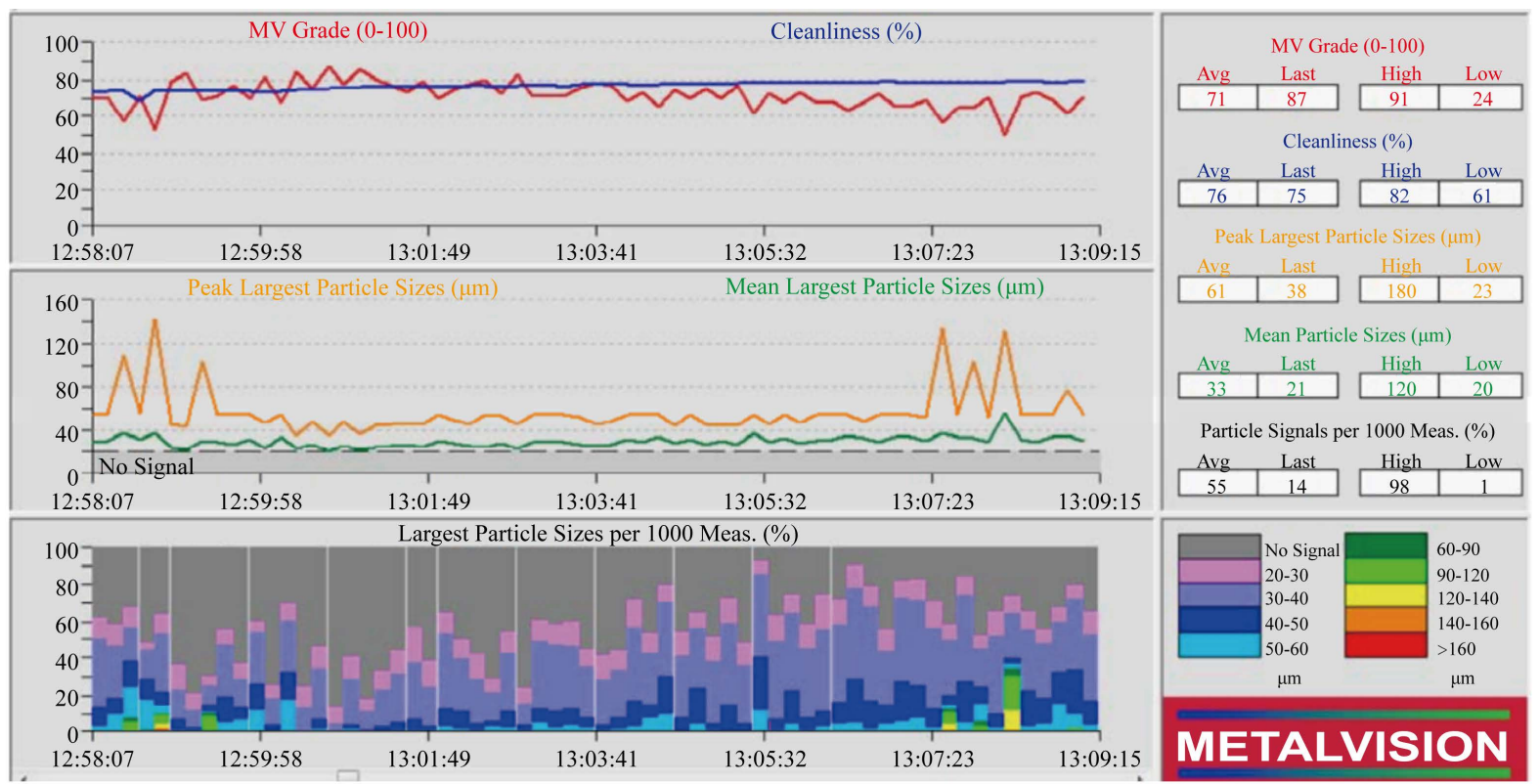

Figure 3. A typical example of inclusion detection result by MetalVision [16]. 


\subsection{Ultrasonic Supported Filtration}

Filtration systems using ultrasonics are usually a combination of a filtering media and an ultrasonic transducer [19]. To prevent particles to directly clog the filter, a multilayered filter, with the size of the mesh decreasing in each layer, is commonly used in the industry. It is obvious that the finer the mesh, the finer the size of the retained particles; but for too many layer or too fine filter, the filtration is impossible due the capillarity pressure required to pass through the filter becomes too great.

Use of ultrasonic waves for the filtration has two main effects. Firstly acoustic streaming can help disperse the sediments at the surface of the filter. Secondly, the cavitation induces at the entry of the capillary allows for the sonocapillarity effect, in which the melt is pushed through the filter channel due to the explosion of the cavitation bubble. This allows the melt to go through multilayered filter even if the capillarity pressure would have been too high to allow filtration [13].

\subsection{Ultrasonic Supported Degassing}

Porosity is one of the major problems in aluminium products, occurring because of the precipitation of hydrogen due to the decrease of its solubility during the solidification. An efficient and fast method for degassing of aluminium melts is based on inducing cavitation in the melt by submitting it to ultrasonic waves. Noble gas is usually blasted through the melt and creates bubbles to remove the inclusions. However, in the case of ultrasonic applications, as the variation of pressure due to the waves occurs, numerous bubbles are created endogenously in the melt, which grow through rectified diffusion and remove the hydrogen while rising up to the surface [20]. An example of the purity of aluminium alloy before and after ultrasonic supported degassing can be seen in Figure 4 [21].

The use of ultrasonic waves in the metallurgical industry is rising, mostly through the improvement of melt treatment it allows. Currently only the MetalVision system offers a solution for detection of particle in the melt using ultrasounds. The potential offered by ultrasonic waves for the detection needs to be deepened.

\section{Prerequisites for a Set-Up for Ultrasonic Detection of Solid Inclusions in Aluminium Melts}

The techniques using ultrasonics are already being used in different fields such as medicine and conditions of ultrasonic applications are well-known. However, the applications in melts need new challenges.

The current status of inclusion detection by ultrasounds allows us to describe the prerequisite for a detection set-up. Three main areas can be described, where the most challenges happen: The piezoelectric element is responsible for the generation of the waves, whose specifications have an influence on the detection. The buffer rod, transmitting the waves to the melt, whose shape and material ensure less signal loss and the contact point between the rod and the melt. Lastly, the particle and melt movement effects the detection, with the influence of particle concentration, settling and melt velocity.

Each area should be investigated in order to optimize the efficiency and sensibility of the detection set-up.

\subsection{Piezoelectric Element Definition}

The piezoelectric element is responsible for creating the ultrasonic waves. However, some key points must be
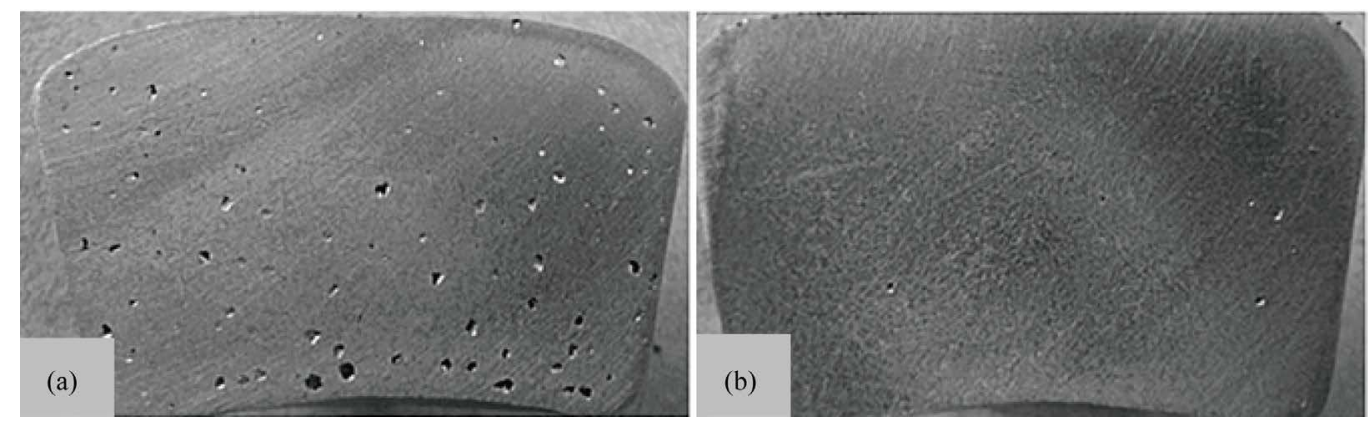

Figure 4. Cross section of aluminium alloy before (a) and after (b) melt treatment by ultrasonic waves [21]. 
investigated to calibrate the detection such as the influence of the amplitude, coupling the transducer to the rod and prevention the unwanted motion (vibration) of the rods.

\subsubsection{Frequency and Amplitude}

Although the theory behind ultrasonics is well developed and understood, no detailed theory has been specifically applied for particle detection in melts. Developing such a theory is primordial to improve the efficiency of the set-up and to find the most suitable specifications for the piezoelectric element.

It appears that the particle detection is a function of the power of the waves rather than its frequency, with a relation between the size of the detected particle and the power of the wave in logarithm:

$$
d B=A-B \log p
$$

where $A$ and $B$ are two constants related to the frequency and the characteristics of the emitting system, and $\mathrm{p}$ the size of the detectable particle [22].

Using high frequency allows for a more focused area of detection and a better discrimination, but it also increases the loss of power inside the melt [23]. Having a more focused area of detection prevents getting the knowledge on a bigger volume of melt. In the past work of Mountford and Sommerville, different particles with known size and shape were aimed to detect by ultrasonic waves. A frequency of $10 \mathrm{MHz}$ was used but the last experiments usually had a frequency of $2.25 \mathrm{MHz}$. The number of the particles inside molten aluminium was measured for 30 seconds at power level between 76 and $88 \mathrm{~dB}$, under stirred and non-stirred conditions. The test set-up was able to detect particles down to $10-15 \mu \mathrm{m}$. The results showed promising data both in water and molten aluminium [22]. It should be noted that using lower frequency will allow detecting the concentration of particles in a bigger melt volume, an advantage over other techniques such as LiMCA, and thus improving the knowledge of the cleanliness of the whole melt.

The measurement procedure during the detection can be summarized as following: sending pulses in the melt, usually at a rate of 100 pulses per second at a given frequency, the amplitude is slowly increased until a reflected signal is received. This first signal gives the size of the largest particle in the melt. This means that, to be able to detect smaller particles, the sound system must be able to emit at a high power level, usually around $90 \mathrm{~dB}$ [22]. This should be taken into account while building the sound system.

\subsubsection{Coupling of the Head and the Buffer Rod}

A main issue of the ultrasonic detection is the temperature range at which transducers can be operated. A piezoelectric element cannot be directly inserted in the melt to emit, so the use of a buffer rod is mandatory. One side of the rod is attached to the emitting element and the other side is inserted into the melt. Without cooling, the transducer can reach more than $160^{\circ} \mathrm{C}$ due to conduction of the heat in the rod, which is beyond the working temperature of such transducers, thus air-cooling is usually used [3] [9]. A thin layer of thermally conductive material can be placed between the rod and the transducer as seen in Figure 5 to prevent overheating [24].

\subsubsection{Loss in Sensitivity Due to Vibration Noises}

The sensitivity of the device is directly related to the Signal-to-Noise Ratio (SNR). It is defined as the ratio between the strength of the desired signal at the end of the probe and the strength of the noise produced inside. This noise is generated due to the mode of vibration and diffraction. It has been shown that the detectable size of the particles depends on the power of the waves rather than the frequency; therefore a higher ratio means better sensitivity of the device as more energy is transferred inside the melt [3] [25].

The maximal power delivered by the piezoelectric element is of primary importance, but studies also show that using a cladding [3] [25] on the rod, and/or using a tapered rod [3], improves the signal guidance and increase the SNR. The cladding also prevents the dissipation of the wave power on the sides of the rods, and thus allows more power transmitted to the melt.

The signal to noise ratio is determinant in the ability of the device to differentiate between different particles when the refracted signal arrive at the same time. It is a very important specification of the rod that has to be taken into account.

\subsection{Probe \& Buffer Rods Definition}

The buffer rod has a primordial importance on the transmission of the ultrasonic waves between the piezoelec- 
tric element and the melt. Its shape, material and coating have influences on the sensitivity of the system.

The design has to be made between two possible configurations: the "Pulse Echo" configuration, which uses a single rod as both transmitter [26] and receiver, and the "Pitch Catch" configuration, in which two rods are used, one as transmitter, one as receiver, as seen in Figure 6.

The Pulse Echo configuration has the advantage of being cheaper and easier to set up, but suffers from higher noise and echoes inside the rod, which decreases the quality of the detection due to the delay line. The Pitch Catch configuration has a better SNR and allows focusing the detection in one particular area by changing the relative position of the rods, but the set-up is more expensive [3] [27]. Despite that increase in cost, the most commonly used configuration is Pitch Catch, as is also allows for a wider area of detection and a better sensitivity.

\subsubsection{Dimensions of the Buffer Rod}

Buffer rods are typically between $200 \mathrm{~mm}$ and $300 \mathrm{~mm}$ in length [3] with a diameter around $15 \mathrm{~mm}$ [27]. This allows for the piezoelectric element to be far enough from the melt and for the rod to be submerged to a depth of around $15 \mathrm{~mm}$, which has been seen sufficient to prevent it from going out of the melt, even with surface level variations due to melt movements [22].

Using a tapered rod is a good solution to prevent echoing inside the buffer rod. Usually an angle around $1.5^{\circ}$ is enough to see considerable improvement in the SNR [3]. Double-tapered rods were designed as shown in

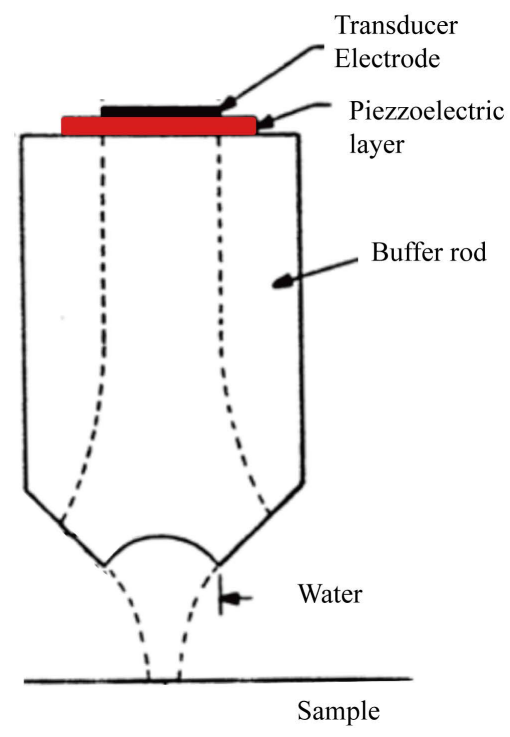

Figure 5. Schematic of a piezoelectric element coupled to a buffer rod.
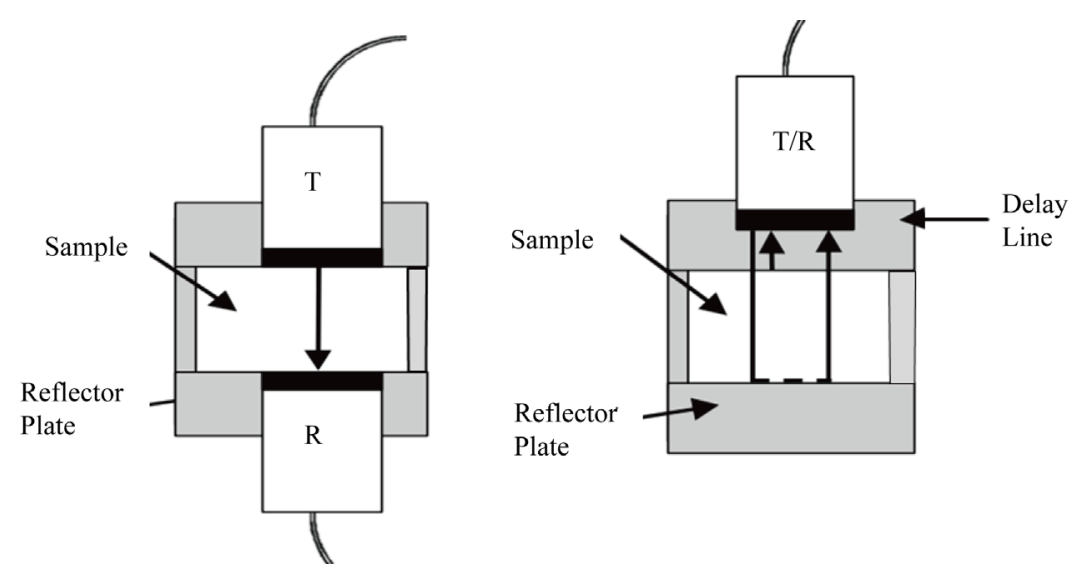

Figure 6. Illustration of the pitch-catch principle (left) and the echo-pulse principle (right). 
Figure 7 to prevent the diameter difference between the start and the end of the rod, allowing both ends to have a diameter around $15 \mathrm{~mm}$ and using an angle up to $2^{\circ}$ [27] [28].

A focusing lens can be used at the end of the rod to increase the power transmitted to a particular area in the melt [28] as shown in Figure 8. This technique allows for the detection of smaller inclusions [9] but reduce the volume of detection in the melt. As the purpose of the ultrasonic technique is to get a wider and faster knowledge of the particle concentration of the melt, using flat ends for the rods is recommended.

\subsubsection{Material Type of the Rod}

Material type is especially important from the metallurgical point of view due to aggressive chemistry of aluminium which is not easily combined with many materials. The choice of material for the rod is of primary importance for the life of the probe, as well as for its effectiveness. A good material should meet the following requirements: good wetting ability by the liquid metal under ultrasonic waves, good acoustic conductivity, good resistance to corrosion, good resistance to thermal shocks, low thermal conductivity and high melting point. The durability of the material can also be improved by using coatings and cooling [13] [29].

Steel rods are mainly used [22] as they provide sufficient acoustic conductivity, but they suffer from corrosion. Good results have also been achieved using Niobium alloys [29]. Other metals can be used, such as silica, tin, alumina or aluminium alloys, but they are not very common [3] [29].

A stainless steel cladding [27] is almost always applied as although it is not the best choice because of aggressivity of aluminium melts. The cladding is applied by thermal spraying. Ceramic cladding was also used in some works [2], but its use is less common. Using a cladding protects the sides of the rod against corrosion.

\subsubsection{Positioning of the Buffer Rods in the Set-Up}

Two types of positioning of the rods are possible: parallel or tilted toward another (V-shape) as shown in Figure 9.

The V-shape setup creates for more focused signals in the melt, thus increasing its sensitivity. The receiving rod receives a higher percentage of the reflected waves and particles down to $10 \mu \mathrm{m}$ can be detected [22]. However, the detection volume is smaller and the system loses the global knowledge as it gains higher sensitivity.

The parallel shape works on the same principle but the waves are transmitted vertically in the melt, and the receiving rod gets a smaller part of the reflected waves. This allows for a bigger volume in the melt to be analysed, but the amplitude of the initial waves has to be higher to be able to detect smaller particles.

It should be noted that one type of set-up might be preferable depending on the aim of the analysis. If the aim is to detect the smallest particle in the melt, using a V-shape with focusing lens at the end of the rod might work better. If the aim is to get a global knowledge of the particle size and concentration in the melt, using a parallel shape system is recommended.

There has been no comparative research done about the influence of the distance between the two rods on the sensitivity of the system. It should be investigated for the further works either.

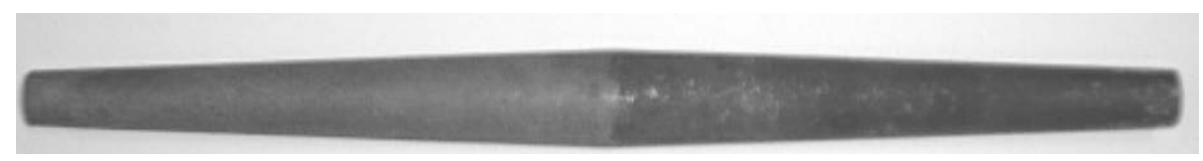

Figure 7. Double tapered rod [27].

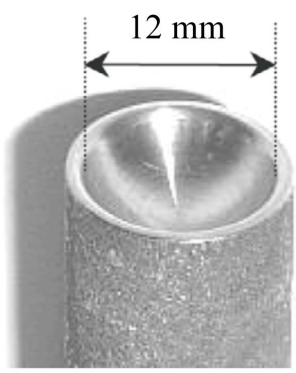

Figure 8. Focusing lens at the end of the rod [27]. 


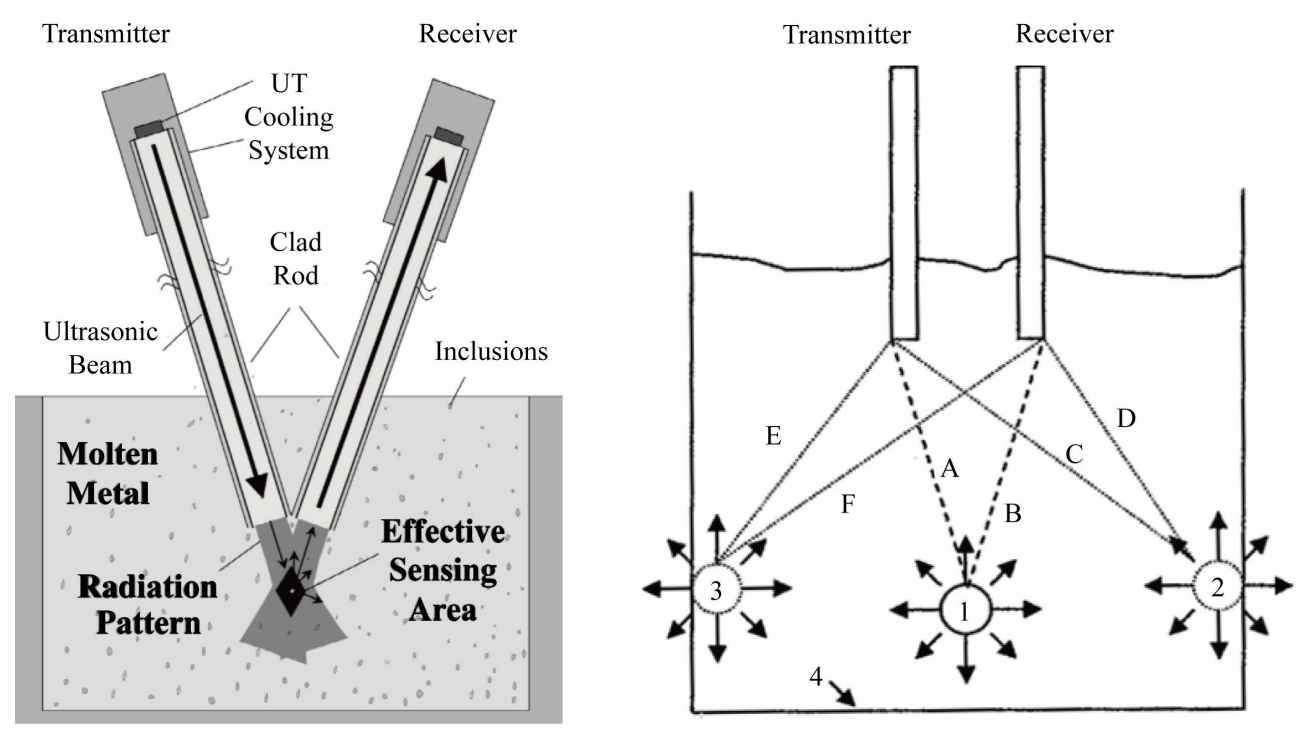

Figure 9. Example of a parallel and V-shaped shape set-up [28].

\subsubsection{Contact Problems between Rods and the Melt}

The set-up for detection suffers from a few recurring problems at the interface between the rod and the melt. Some corrosion may occur and a cleaning can be needed to increase the lifespan of the rod.

First problem is the coating around the steel rod does not protect the tip from the corrosion. A typical AlFe3 alloy deposit has been found on the tip of the rod in the work of Mountford after a long period of continuous detection [22].

The second problem, which can be faced in both laboratory and industrial utilizations, comes from the tendency of particle to gather at the tip of the rod [30]. The particles decrease the transmission of the waves by covering the tip and this impacts the efficiency of the detection [3]. A mechanical scratching of the tip is usually performed; either automated or manual solutions have been found [23]. An automated scratching is of premium importance, as it can be done more frequently without human intervention.

\subsection{Particle and Melt Movement Effects on Detection Efficiency}

Interactions between melt and particles must be also well understood in terms of turbulence, settling and agglomeration. These phenomena affect the detectable particle size range, detectable volume and the reliability of the detection results.

\subsubsection{Settling Effect on Detectable Particle Size Range}

The modulation of the amplitude of the received signal gives an indication of the site, and the first particles detected are the biggest ones [23]. But some problems may occur due to different settling velocities of particles. The big particles settle rapidly and they move out of the detection zone. Their accumulation on the bottom of the furnace creates noise and impact the detection procedure. This problem can be solved by stirring the melt which carries the settled particles again in the detectable volume, but the stirring should not occur during the detection, rather just before, as stirring can artificially increase the number of detected particles [22].

In the work of Mountford, pure aluminium at about $730^{\circ} \mathrm{C}$ was used for the ultrasonic detection and alumina particles were added and detected for 30 seconds with waves of $2.25 \mathrm{MHz}$ at $72 \mathrm{~dB}$ to observe the detecting process. By addition of alumina with known concentration, the number of the detected particles was expected to increase linearly however it was not observed as it is seen in Figure 10. These irregularities in the results could have been caused by the irregular shape of $\mathrm{Al}_{2} \mathrm{O}_{3}$ particles [22]. This result brings the question if the detectable size also might be influenced by the shape of the particles.

However, the shape factor has also a direct influence on settling velocity of particles which can cause different problems during the detection [31] [32]. The shape of the particles could have been a reason for unpromising results but more research is needed in this topic. 


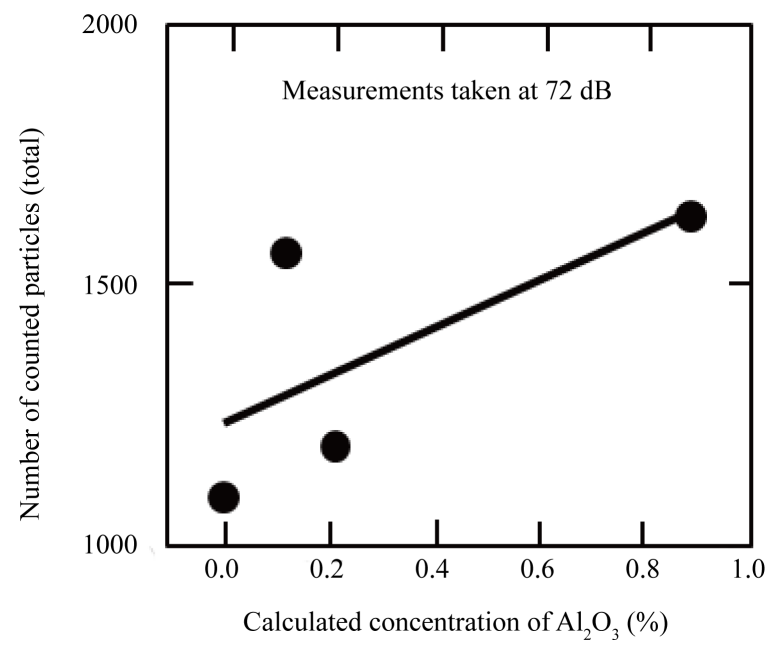

Figure 10. Variation of the detected particle concentration with added alumina concentration.

\subsubsection{Melt Flow Effect on Detection}

Ultrasonic detection can potentially be used in launder, holding furnaces and crucible furnaces. Slower melt velocities such as in crucible or holding furnaces make the calibration of over counting particles easier. However, it has been reported by M. Badowski et al. that the melt velocity can disturb the particle movement even in crucible furnaces [32]. But high turbulence such as in the case of launders can make the calibration more complicated but detection can be still performed.

Stirring also affects the melt flow and the detection characteristics since it prevents the settling and keeps the big particles in the flow [22].

\subsubsection{Particle Concentration Effect on Detection}

The use of water model is a good way to test theory on the minimal detectable size of the particles. The usual minimal size is around $20 \mu \mathrm{m}$ [22], which is also the minimal detectable size by MetalVision. Detection of the particles down to $10 \mu \mathrm{m}$ is theoretically possible by focusing the zone of detection, using a V-shape system. However, there are some other influences which can contradict the theoretical predictions.

The sensibility of the detection is affected by the concentration of particles in the melt. For high inclusion concentrations, the particle count is often less than the real because some of the small particles are hidden in the "shadow" of the bigger particles. A big percentage of particles are not reachable by the reflected ultrasonic waves [23]. This problem occurs also in lower concentrations but the influence is statistically not important. Stirring the melt is a solution for this, as it will make the particle move out the shadow of big particles, and it is possible to correct the count by calculating the average number of detected particles [22]. But this solution also creates new problems due to a melt movement. In industrial applications, this problem usually doesn't occur since the particle concentration is very low.

\section{Conclusions}

The previous research in the years 1985 to 2015 on ultrasonic particle detection in aluminium melts can be summed up as follows:

- An on-line monitoring of inclusions would allow for faster and more efficient processes.

- The physics of ultrasonic is well-known and allows creating and arranging models. Existing water models can be used for the first tests to validate the setup.

- MetalVision is expensive equipment, which has limits when the contrast between concentration of bigger sized particles and smaller sized particles is too high. This could be improved by developing a new on-line system.

- A set-up for detection by ultrasonic waves might be described in 4 parts: the piezoelectric transducer in charge of producing the waves, the rods which are transmitting the waves to the melt, the interface between 
the rods and the melt, and finally the interface between the waves inside the melt and the particles.

- The detection of inclusions in aluminium melts by ultrasonic waves seems promising, and should be experimented more on an industrial level to see whether the change of scale has an influence on the results.

\section{Future Prospective}

- This work is a review of different problematics and possibilities associated with the particle detection by ultrasonic waves. Although it is working both theoretically and on a laboratory scale, more tests are surely needed.

- Future works should focus on combining different futures of ultrasonic detection such as detection of the size of the particles, their position in the melt and the concentration of the melt. The question still stands if it is possible to do all of these together.

- Another area for the future research would be improving the rod. Finding a compromise between good conductivity and corrosion resistance is very important which would make the detection system cheaper than the current solutions.

\section{Acknowledgements}

The research leading to these results has been carried out within the framework of the AMAP (Advanced Metals and Processes) research cluster at RWTH Aachen University, Germany.

\section{References}

[1] Dispinar, D. (2005) Determination of Metal Quality of Aluminium and Its Alloys. University of Birmingham, Birmingham.

[2] Kurban M., Sommerville I.D., Mountford N.D.G. and P.H. Mountford (2005) An Ultrasonic Sensor for the Continuous Online Monitoring of the Cleanliness of Liquid Aluminium. Light Metal 2005, TMS San Francisco February 2005, 945-949.

[3] Poynton, S., Brandt, M. and Grandfield, J. (2009) A Review of Inclusion Detection Methods in Molten Aluminium. In: Light Metals, Vol. 3, John Wiley \& Sons, Hoboken, 681-687.

[4] Currie, A.J., Millman, S. and Johnstone, S. (2009) An Ultrasonic Liquid Steel Monitor: Measurement Principle and Probe Design. AIP Conference Proceedings, 1096, 1735-1742. http://dx.doi.org/10.1063/1.3114168

[5] ABB-Brochure for PoDFA.

[6] ABB-Brochure for LiMCA.

[7] Smith, D.D., Hixson, B., Mountford, H. and Sommerville, I. (2015) Practical Use of the Metal Vision Ultrasonic Inclusion Analyzer. TMS Orlando.

[8] Gökelma, M., Morscheiser, J., Badowski, M., Dang, T., Le Brun, P. and Tewes, S. (2015) Observation on Inclusion Settling by LiMCA and PoDFA Analysis in Aluminium Melts. International Aluminium Journal, 91, 56-61

[9] Ihara, I., Aso, H. and Burhan, D. (2004) In-Situ Observation of Alumina Particles in Molten Aluminum Using a Focused Ultrasonic Sensor. JSME International Journal Series A, Solid Mechanics and Material Engineering, 47, 280286

[10] Friedrich, B. and Kräutlein, C. (2006) Melt Treatment of Copper and Aluminium—The Complex Step Before Casting. IME Process Metallurgy and Metals Recycling, RWTH Aachen, Aachen.

[11] Bao, S. (2011) PhD Thesis "Filtration of Aluminium—Experiments, Wetting, and Modelling". Norwegian University of Science and Technology, Trondheim.

[12] Mansfield, T.L. (1982) Ultrasonic Technology for Measuring Molten Aluminium Quality. Journal of Metals, 34, 5457.

[13] Eskin, G.I. and Eskin, D.G. (2015) Ultrasonic Treatment of Light Alloys Melt. CRC Press, Boca Raton.

[14] Auld, B.A. (1990) Acoustic Fields and Waves in Solids. Vol. I \& II, 2nd Edition, Krieger Publishing Company, Malabar.

[15] Lecture Notes of the University of Washington. Department of Bioengineering. Seattle.

[16] Metal Vision Manufacturing Canada Ltd., Brochure.

[17] Neff, D.V. (2004) Evaluating Molten Metal Cleanliness for Producing High Integrity Aluminium Die Castings. Die Casting Engineer, 48, 24-30. 
[18] Guo, J.F. (2007) The Use of Ultrasonic Techniques in Measuring Inclusions in Al-Si Alloys Melts. Université du Quebec à Chicoutimi, Chicoutimi.

[19] Vadoothker, A.R. (2001) Ultrasound-Assisted filtration System. Patent US6221255 B1.

[20] Eckert, C.E. (1998) Apparatus and Method for Ultrasonic Particle Detection in Molten Metal. Aluminum Company of America, United States Patent US4563895 A.

[21] Puga, H., Barbosa, J., Gabriel, J., Seabra, E., Ribeiro, S. and Prokic, M. (2011) Evaluation of Ultrasonic Aluminium Degassing by Piezoelectric Sensor. Journal of Materials Processing Technology, 211, 1026-1033. http://dx.doi.org/10.1016/j.jmatprotec.2011.01.003

[22] Mountford, N.D.G., Simionescu, A. and Sommerville, I.D. (1997) Visualization of the Particle Content of Liquid Aluminium Alloys. Light Metals, 937-943.

[23] Sommerville, I.D., Mountford, N.D.G. and Martins, L.C.B. (2000) Laboratory and Industrial Validation of an Ultrasonic Sensor for Cleanliness Measurement in Liquid Metals. Light Metals, 721-726.

[24] Spicci, L. and Vigna, G. (2014) Heat Drain Device for Ultrasound Imaging Probes. Proceedings of the 2014 COMSOL Conference in Cambridge, Cambridge, 17-19 September 2014.

[25] Jen, C.K., Legoux, J.G. and Parent, L. (2000) Experimental Evaluation of Clad Metallic Buffer Rods for High Temperature Ultrasonic Measurements. NDT \& E International, 33, 145-153. http://dx.doi.org/10.1016/S0963-8695(99)00042-0

[26] Ono, Y., Moisan, J.F., Zhang, Y., Jen, C.K. and Su, C.Y. (2004) An On-Line Ultrasonic Cleanliness Analyzer for Molten Light Metals. JOM, 56, 59-64. http://dx.doi.org/10.1007/s11837-004-0148-9

[27] Ono, Y., Moisan, J. F., Jen, C.K. and França, D.R. (2002) Development of Ultrasonic Techniques with Buffer Rod in Molten Aluminum. Ultrasonics Symposium, 2002, Proceedings, 2002 IEEE, 1, 805-810.

[28] Ono, Y., Moisan, J.F. and Jen, C.K. (2003) Ultrasonic Techniques for Imaging and Measurements in Molten Aluminium. IEEE Transactions, 50, 1711-1721.

[29] Mansfield, T.L. (1981) Probe for the Ultrasonic Inspection of Molten Aluminium. Reynolds Metals Company, United States Patent US4261197 A.

[30] Gallo, R., Mountford, H. and Sommerville, I. (2003) Ultrasound for On-Line Inclusion Detection in Molten Aluminium Alloys: Technology Assessment. AFS International Conference on Structural Aluminium Castings, Orlando, 2-4 November 2003, 179-194.

[31] Gökelma, M., Le Brun, P., Dang, T., Badowski, M., Morscheiser, J., Friedrich, B. and Tewes, S. (2016) Assessment of Settling Behavior of Particles with Different Shape Factors by LiMCA Data Analysis. Light Metals.

[32] Badowski, M., Gökelma, M., Morscheiser, J., Dang, T., Le Brun, P. and Tewes, S. (2015) Study of Particle Settling and Sedimentation in a Crucible Furnace. Light Metals, 967-972. 